\title{
License Plate Recognition System using Neural Networks and Multithresholding Technique
}

\author{
Bharat Bhushan \\ M.Tech. Scholar \\ Deptt. of Electronics and \\ Communication \\ Chandigarh Group of Colleges, \\ Landran
}

\author{
Simranjot Singh \\ B.Tech. Scholar \\ Deptt. of Computer Science \\ Guru Nanak Dev University, \\ Regional Campus, Jalandhar
}

\author{
Ruchi Singla, Ph.D \\ Associate Professor \\ Deptt. of Electronics and \\ Communication \\ Chandigarh Group of Colleges, \\ Landran
}

\begin{abstract}
License plate recognition is a fully automated real time technique that has been widely used for identification, theft control and security validation of vehicles. For recognition and extraction of desired regions of the number plate of the vehicle, different algorithms are used. An image processing technology based on license plate recognition (LPR) that is being used to identify vehicles, using neural networks and image co-relation was developed by K. Yilmaz [2]. In this paper, a different novel approach has been presented to increase the quality of the image and to enhance the results for extracting license plate from dull and low intensity images. In the previous technique the recognition rate (percentage of image recognized) reached was $96.64 \%$ [2], but now using multithresholding and neural pattern recognition (NPR) techniques together with artificial neural networks, a higher recognition rate of $98.40 \%$ has been achieved. Certain problems related to neural networks in the previous research methodology such as blobs extraction, segmentation and character recognition, that inhibit complete extraction of features from number plate of the vehicle were analyzed in this approach. The proposed technique helps to improve the quality of the images and detect the characters or digits of the number plate with a better recognition rate.
\end{abstract}

\section{General Terms}

License plate recognition, Multithresholding Technique, Neural Pattern Recognition, Artificial Neural Networks.

\section{Keywords}

LPR, Feature Extraction, Multithresholding.

\section{INTRODUCTION}

Day by day license plate recognition (LPR) systems are becoming an important safeguard measure to implement video monitoring for community security [1]. For intelligent video monitoring and processing, the key content is automated license recognition. A license plate recognition system uses unique identification number which is assigned to each car by administrative authorities. Technically vehicle identification here is an application of image processing technique. Automated image recognition has a wide range of applications such as criminal pursuit, community monitoring, vehicle status checking, automatic toll collection and traffic flow magnitude control [4].

The Practical implementation of intelligent transport system by using camera is a very exacting process since the system has to form dynamic image in an environment where pollution impacts the license plate along with actual road conditions and camera installation angle variations [1]. All of these problems bring difficulty in character recognition by a LPR system, but by implying the neural networks theory in LPR systems researchers are able to position and recognize them more effectively.

\section{OVERVIEW}

\subsection{Related Work}

A license plate recognition system generally follows four steps: image capturing, image processing, character segmentation and character recognition. Image correlation and character segmentation methods were used in previous work. In [1-3] a fuzzy based method was introduced using neural networks. The work presented in [2] is a smart hybrid approach using neural network and image correlation technique and is applied for recognizing numbers and characters from the number plate of the vehicle. Research papers $[13,3,10,14,2]$ express the work done on neural networks and show how the recognition rate of the vehicle license plate goes better day by day.

\subsection{System Overview}

License plate detection and recognition attract many researchers to search and develop systems which can process images and obtain useful information from them. Hence, the segmentation problem is the main limitation of the previous research work done. Several techniques have been used to increase the recognition rate such as artificial neural networks, automatic recognition and character recognition.

\section{METHODOLOGY}

In the proposed research, multithresholding and neural pattern recognition (NPR) techniques are used. At first, a black and white image of license plate is captured. Now, this captured image is converted to a gray scale image. Gray scale conversion is done to highlight the white area in the captured image since the unwanted noise has to be removed with the median filter.

As the noise is removed the image intensity and the background of the image become more clear, following which histogram equalization and contrast stretching method is applied in the license plate. Histogram equalization is applied to observe exact white level pixel background intensity of captured license plate after the removal of noise. Contrast stretching method is applied on the detected image in order to increase the white level pixel background intensity. Multilevel thresholding is applied to maintain equal space between the digits and alphabets. Finally, we used artificial intelligence in terms of neural networks that automatically detected the captured image. Now to train the neural network we followed three steps: neural training, validation and testing as demonstrated in Figure 1.1. 


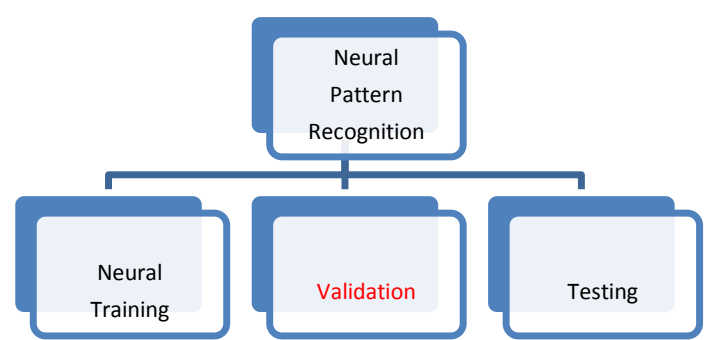

Fig 1: Neural pattern recognition methodology

\section{SYSTEM BLOCK DIAGRAM}

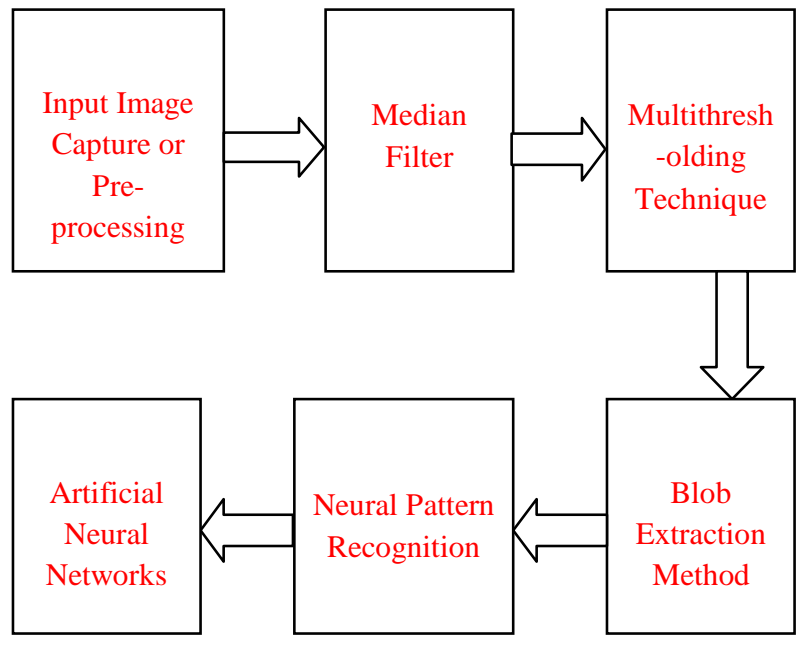

Fig 2: Basic Block Diagram of License Plate Recognition

\subsection{Pre-processing}

In the pre-processing method, firstly the captured image is converted to gray scale image. This method is applied to make the background of the image more clear that is white.

\subsection{Median filter}

In order to filter out the unwanted portion of the image and replace it with a white background, we need a filter mechanism. Median filter is applied to remove this unwanted noise from the image, that eventually improves the recognition rate.

\subsection{Multithresholding Technique}

For image segmentation, the most powerful technique is multithresholding. The application of multithresholding techniques is based on the assumption that object and background pixels in a digital image can be distinguished by their gray-level or color values. The proposed multithresholding technique can be applied to gray-level images as well as to color images.

\subsection{Blob extraction method}

Blob extraction method is commonly used to remove the segmentation problem so that the characters or digits in the number plate are not combined together. This method is very helpful in finding the proper intensities of each digit or alphabet.

\section{blob no:1 blobno:2 blob no:3 blobno:4 blob no:5

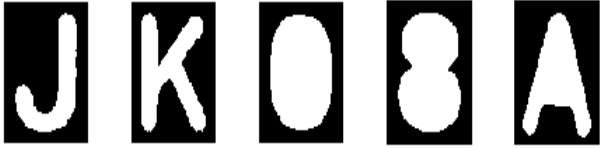

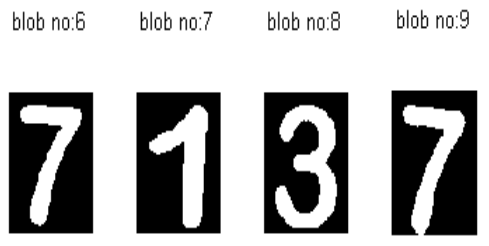

Fig 3: Blob Extraction Method

\subsection{Pattern Recognition}

The act of recognition can be divided into two broad categories: recognizing concrete items and recognizing abstract items. The recognition of concrete items involves the recognition of spatial and temporal items (PR). Examples of spatial items are fingerprints, weather maps, pictures and physical objects. Examples of temporal items are waveforms and signatures. Recognition of abstract items involves the recognition of a solution to a problem, an old conversation or argument etc. or simply recognizing items that do not exist physically.

\subsection{Feature Extraction}

Feature extraction is a process of studying and extracting useful information from the filtered input patterns. The derived information may be general features, which are evaluated to ease further processing. For example, in image recognition, the extracted features will contain information about the gray shade, texture, shape or context of the image. This is the main information used in image processing. Again, the methods of feature extraction and the extracted features are application dependent [7].

\subsection{Character Recognition}

This is the main step of the system where segmented characters are recognized. Character recognition is also called optical character recognition (OCR). Earlier methods used for recognizing number plate was formula based recognition which has been replaced by neural networks nowadays [7].

\subsection{Artificial Neural Networks}

The pattern recognition approaches discussed so far are based on direct computation through machines. Direct computations are based on math-related techniques and discusses bionicsrelated concepts in recognizing patterns. An artificial neural network is an interconnected group of nodes, akin to the vast 
network of neurons in a brain. Here, in Figure 2.2 each circular node represents an artificial neuron and an arrow represents a connection from the output of one neuron to the input of another.

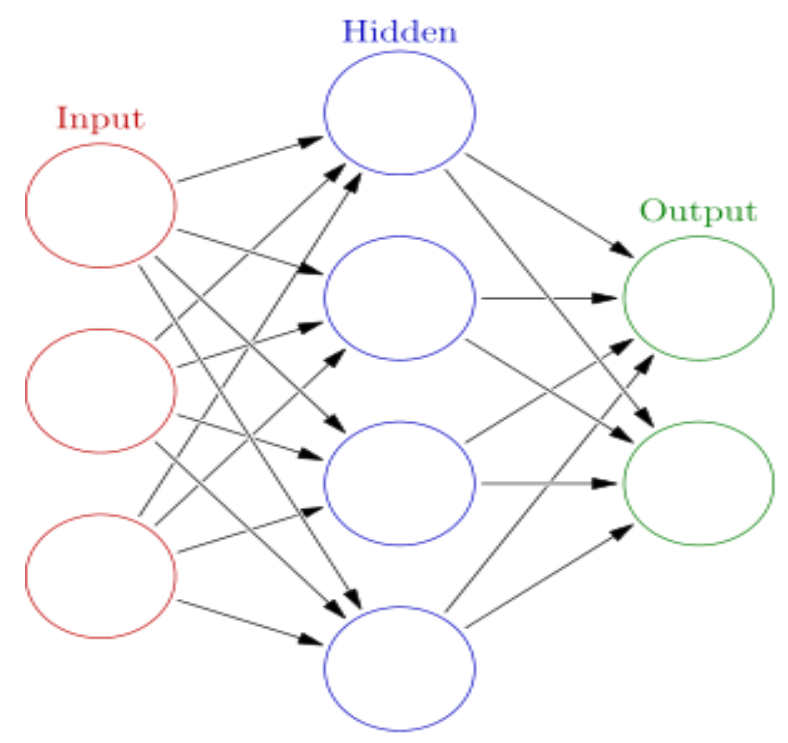

Fig 3: Model of an Artificial Neural Network

\section{LPR FLOW CHART}

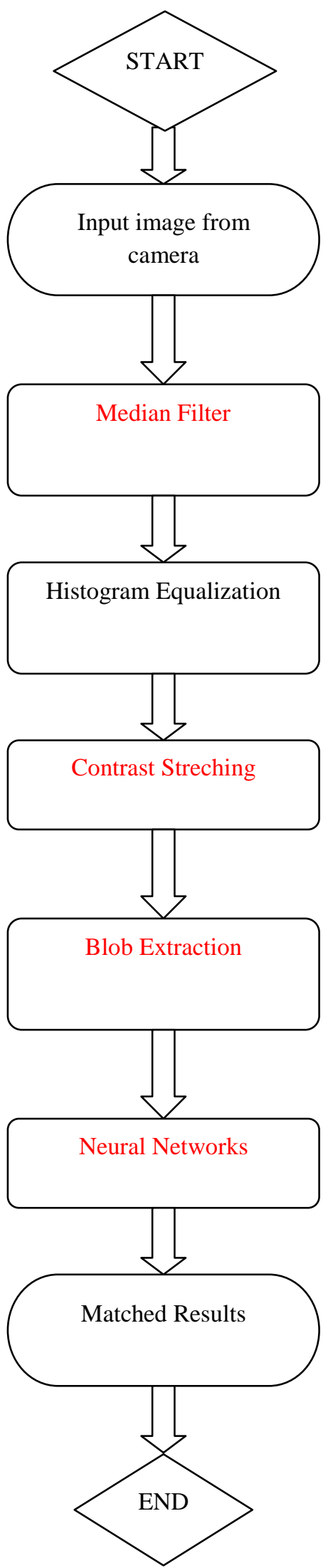

Fig 4: License Plate Recognition flow chart 


\section{ADVANTAGES OF LICENSE PLATE RECOGNITION}

\subsection{Traffic laws regulation}

License plate recognition technology is advantageous in regulation of the traffic rules. For example, a license plate recognition device can be put at an intersection and record the license plate of a car that runs a red light. A citation can then be sent to the registered owner of the car. Similarly, an LPR device can be placed in an area where speeding is a common problem and record the infraction for follow-through with a citation [4].

\subsection{Law Enforcement}

License plate recognition technology has several applications in areas of law enforcement. An LPR device can be mounted on a patrol car to record plates of passing cars. This information can then be compared with the law enforcement data base for vehicles associated with a crime. This is useful in Amber alerts, finding stolen vehicles and executing felony warrants.

\subsection{Vehicle Access}

An LPR system is also useful for vehicle access. On toll roads, these systems can be used to allow cars to pass through toll gates without stopping. The LPR records the plate of the car and associates the number with the registered owner. The bill can then be sent by mail. This technology also can be used in secure, gated locations. When a vehicle recorded in a database approaches a security gate, the system recognizes the license plate, and the gate opens automatically. This allows the driver to pass without interruption and eliminates the possibility of an entrance code being stolen.

\section{APPLICATIONS OF LICENSE PLATE RECOGNITION}

License plate re There are several applications of automatic license plate recognition. The two major values license plate recognition adds to systems are automation and security.

An Intelligent Transportation System equipped with LPR can provide:

1. Flexible and automatic highway toll collection systems

2. Analysis of city traffic during peak periods

3. Automation of weigh-in-motion systems

4. Enhanced vehicle theft prevention

5. Effective law enforcement

6. Effective enforcement of traffic rules

7. Highest efficiency for border control systems, etc.

\section{RESULTS}

The new proposed technique that is using multithresholding with neural networks can recognize the number plates of the vehicles with better recognition rate i.e. with $98.40 \%$. Figure 5 shows the original number plate that has been extracted for the recognition purpose.

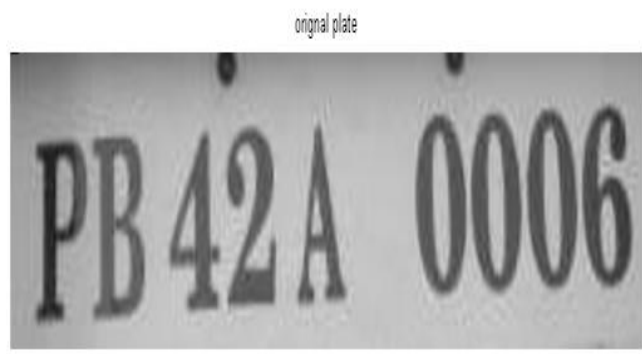

Fig 5: Original number plate

In Figure 6 median filter is applied to remove the noise or unwanted material from the number plate.

plate ater apolying median flter

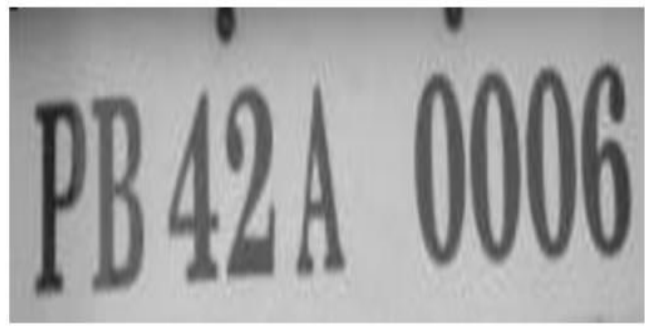

Fig 6: After the application of media filter

Figure 7 shows the histogram equalization results of the detected number plate before contrast stretching. The graph defines the exact received intensity of index values ranges from

50-150.

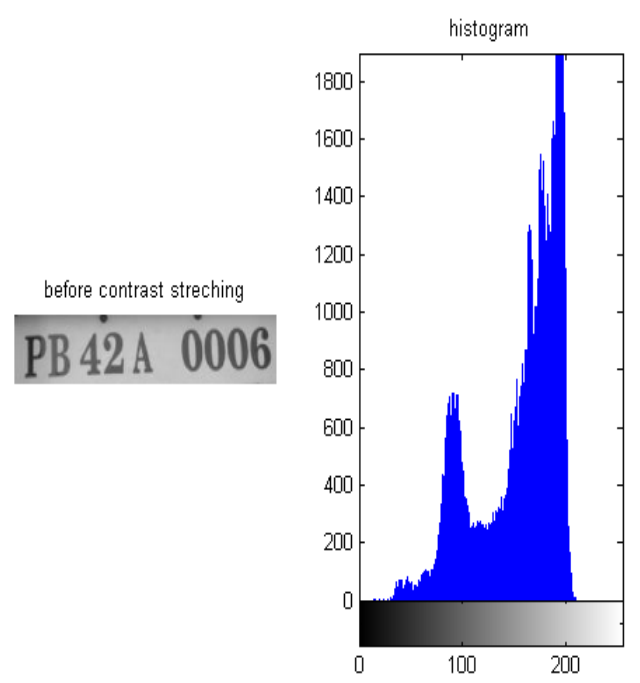

Fig 7: Before contrast stretching histogram

Figure 8 shows the results of the detected number plate after contrast stretching. The graph shows the increased intensity of the white pixels from 50-250. 
after contrast streching PB 42A 0006

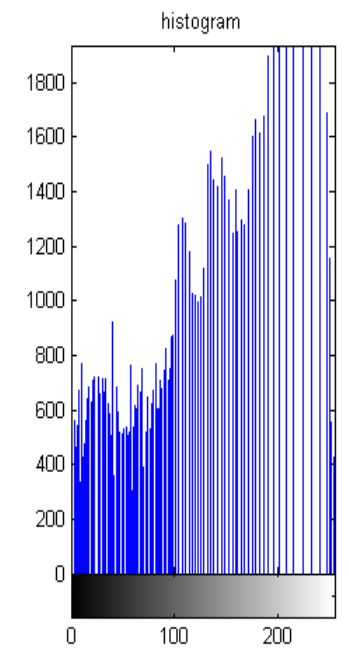

Fig 8: After contrast stretching histogram

\section{OUTPUT IMAGE COMPARISON OF THE PREVIOUS OTSU METHOD \\ AND THE MULTITHRESHOLDING METHOD}

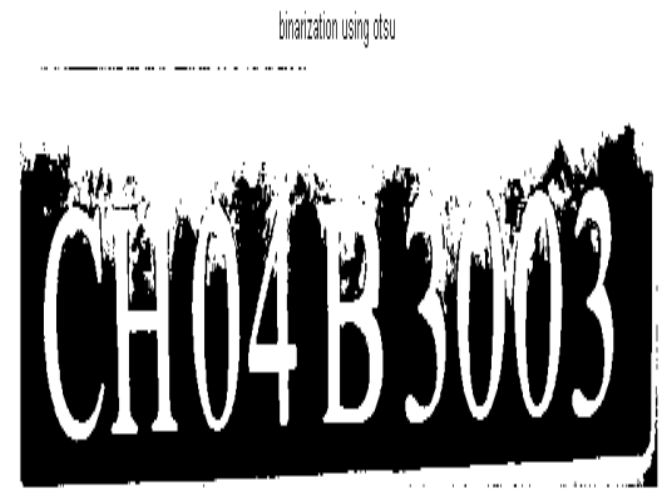

Fig 9: Binarisation using OTSU method with recognition rate of $96.64 \%$

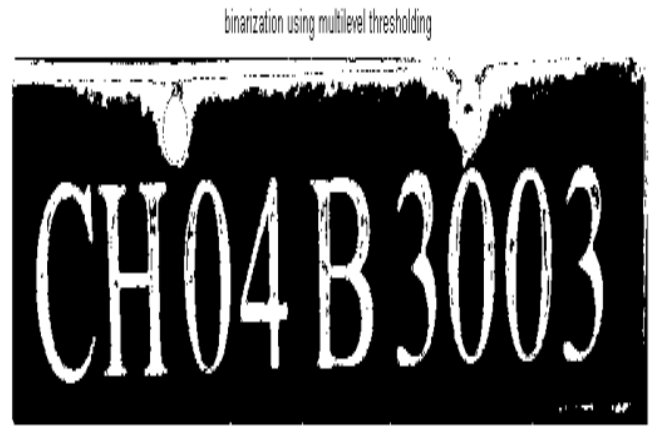

Fig 10: Binarization using the proposed Multilevel Thesholding with increased recognition rate of $98.40 \%$

\section{GRAPHICAL COMPARISON OF PROPOSED MULTITHRESHOLDING TECHNIQUE WITH NEURAL NETWORKS WITH IMAGE \\ CORRELATION}

In Figure 10 the black color bar shows the results of previous technique that is neural networks with image correlation. The white bar in the graph evince the increased recognition rate of $98.40 \%$ from $94.64 \%$ with use of multithresholding with neural networks.

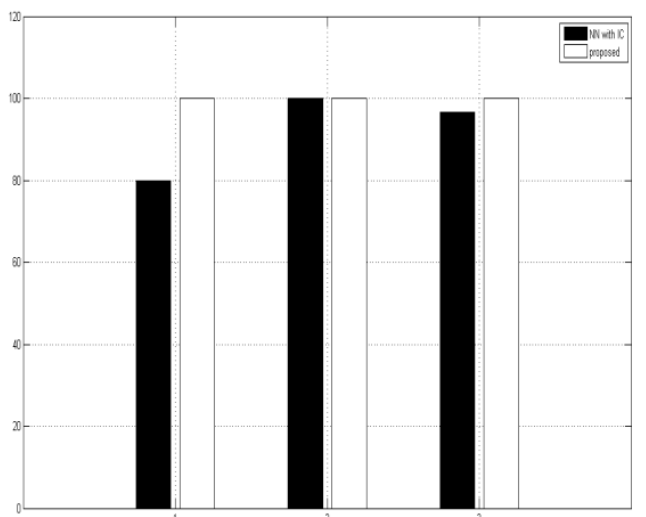

Fig 10: Performance checking graph

\section{CONCLUSION}

This paper compared and analyzed different LPR systems in terms of their recognition rate and discussed different problems faced by other license plate recognition systems. The multithresholding methodology for image segmentation has been introduced in order to recognize license plates with enhanced precision. This hybrid algorithm uses the features of both multithresholding and neural pattern recognition that provided recognition rate of $98.40 \%$. Consequentially the proposed technique is very helpful in realization of license plates at an augmented level of certainty.

\section{REFERENCES}

[1] Ying Wen, Yue Lu, Jingqi Yan, Zhenyu Zhou, Karen M. von Deneen and Pengfei Shi, "An Algorithm for License Plate Recognition Applied to Intelligent Transportation System", IEEE, transactions on intelligent transportation system,vol 12, no 3,sept 2011.

[2] K. Yilmaz ,"A Smart Hybrid License Plate Recognition System Based on Image Processing using Neural Network and Image Correlation",Innovations in Intelligent Systems and Applications (INISTA), 2011 International Symposium on 18 June 2011

[3] Li Li , Feng Guangli "The License Plate Recognition System Based on Fuzzy Theory and BP Neural Network", IEEE,Intelligent Computation Technology and Automation (ICICTA), International Conference on (Volume:1 ) 2011

[4] Lingxian Yang, Heping Chen, Wei Zhang,"A License Plate Recognition Algorithm for Community Monitor",IEEE, Intelligent Networks and Intelligent 
Systems (ICINIS), 2012 Fifth International Conference on,3 Nov. 2012

[5] Chuin-Mu Wang, Ching-Yuan Su,"Fast License Plate Location and Recognition Using Wavelet Transform in Android" ,IEEE, Industrial Electronics and Applications (ICIEA), 7th IEEE Conference on 18-20 July 2012

[6] Yuh-Rau Wangi,Wei- Hung Lin,Ling Yang," novel DSP based Real time license plate detection Algorithm" IEEE, Machine Learning and Cybernetics (ICMLC), International Conference on (Volume:5) 15-17 July 2012

[7] Lorita Angeline, Wei Yeang Kow, Wei Leong Khong, Mei Yeen Choong, Kenneth Tze Kin Teo,"License Plate Character Recognition via Signature Analysis and Features Extraction" 2 IEEE, Computational Intelligence, Modelling and Simulation (CIMSiM),Fourth International Conference on 25-27 Sept. 2012

[8] Aissa Boudjella, Brahim Belhaouari Samir, H. Bt.Daud,Raja Syahira, "License Plate Recognition Part II: Wavelet Transform and Euclidean Distance Method" ,IEEE, Intelligent and Advanced Systems (ICIAS), 4th International Conference on (Volume:2 )12-14 June 2012

[9] Feng Li,License plate recognition algorithm based on fuzzy inference for binary",IEEE, Computational and Information Sciences (ICCIS), Fourth International Conference on17-19 Aug. 2012

[10] Song Qing-kun , Yuan Hui-jun , Zhou Teng,"License Plate Recognition Based On Mathematical Morphology Method and RBF Neural Network", IEEE, Measurement, Information and Control (MIC), 2012 International Conference on (Volume:2 )18-20 May 2012

[11] Jing-Ming Guo, Yun-Fu Liu and Chih-Hsien Hsia,"Multiple License Plates Recognition System",IEEE,System Science and Engineering (ICSSE), International Conference on June 30 2012-July 22012

[12] Jianyu Zhao, Shujian Ma, Weimin Han, Yang Yang, Xudong Wang,"Research and Implementation of License
Plate Recognition Technology",IEEE, Control and Decision Conference (CCDC), 24th Chinese 23-25 May 2012

[13] Wang Yutao, Qin Tingting, Tian Ruixia, Yang Gang,"Recognition of License Plate Character Based on Wavelet Transform and Generalized Regression Neural Network" IEEE, Control and Decision Conference (CCDC), 24th Chinese 23-25 May 2012

[14] Huang Lin , Yang Tie-jun,"Vehicle license plate Recognition Based on Wavelet Transform Modulus Maxima and BP Neural Network", IEEE, Natural Computation (ICNC), Eighth International Conference on 29-31 May 2012

[15] Rong-Tsai Lee , King-Chu Hung,"Real-Time Vehicle License Plate Recognition Based on 1-D Discrete Periodic Wavelet Transform", IEEE, Computer, Consumer and Control (IS3C), International Symposium on 4-6 June 2012

[16] Bo Li, Bin Tian, Qingming Yao, Kunfeng Wang,“A Vehicle License Plate Recognition System Based on Analysis of Maximally Stable Extremal Regions",IEEE, Networking, Sensing and Control (ICNSC), 9th IEEE International Conference on 11-14 April 2012

[17] Xiuxia $\mathrm{Yu}$, Hongyu Cao, Haidong Lu,"Algorithm of License Plate Localization Based on Texture Analysis", IEEE, Transportation, Mechanical, and Electrical Engineering (TMEE), International Conference on 16-18 Dec. 2011

[18] FENG Zhifan , FANG Kangling,"Research and Implementation of an Improved License Plate Recognition Algorithm",IEEE, Biomedical Engineering and Informatics (BMEI), 4th International Conference on (Volume:4 )15-17 Oct. 2011.

[19] Amir Hossein Ashtari, Md. Jan Nordin, Seyed Mostafa Mousavi Kahaki, “A New Reliable Approach for Persian License Plate Detection on Colour Images",IEEE, Electrical Engineering and Informatics (ICEEI), International Conference on 17-19 July 2011 